\title{
The intrinsic phenomena of concentration and cavitation on the Riemann solutions for the perturbed macroscopic production model
}

\author{
Yunfeng Zhang ${ }^{1}$ and Meina Sun ${ }^{1}$ \\ ${ }^{1}$ Ludong University
}

January 31, 2021

\begin{abstract}
The exact solutions of the Riemann problems for the two different perturbed macroscopic production models are considered and constructed respectively for all the possible cases. It is found that the asymptotic limits of solutions to the Riemann problem for the first kind of perturbed macroscopic production model do not coverage to those of the pressureless gas dynamics model, because the delta shock wave in the limiting situation has different propagation speed and strength from those for the pressureless gas dynamics model. In order to remedy it, the second kind of perturbed macroscopic production model is proposed, whose asymptotic limits of Riemann solutions are identical with those of the pressureless gas dynamics model.
\end{abstract}

\section{Hosted file}

Zhang and Sun.pdf available at https://authorea.com/users/392908/articles/506657-theintrinsic-phenomena-of-concentration-and-cavitation-on-the-riemann-solutions-for-theperturbed-macroscopic-production-model

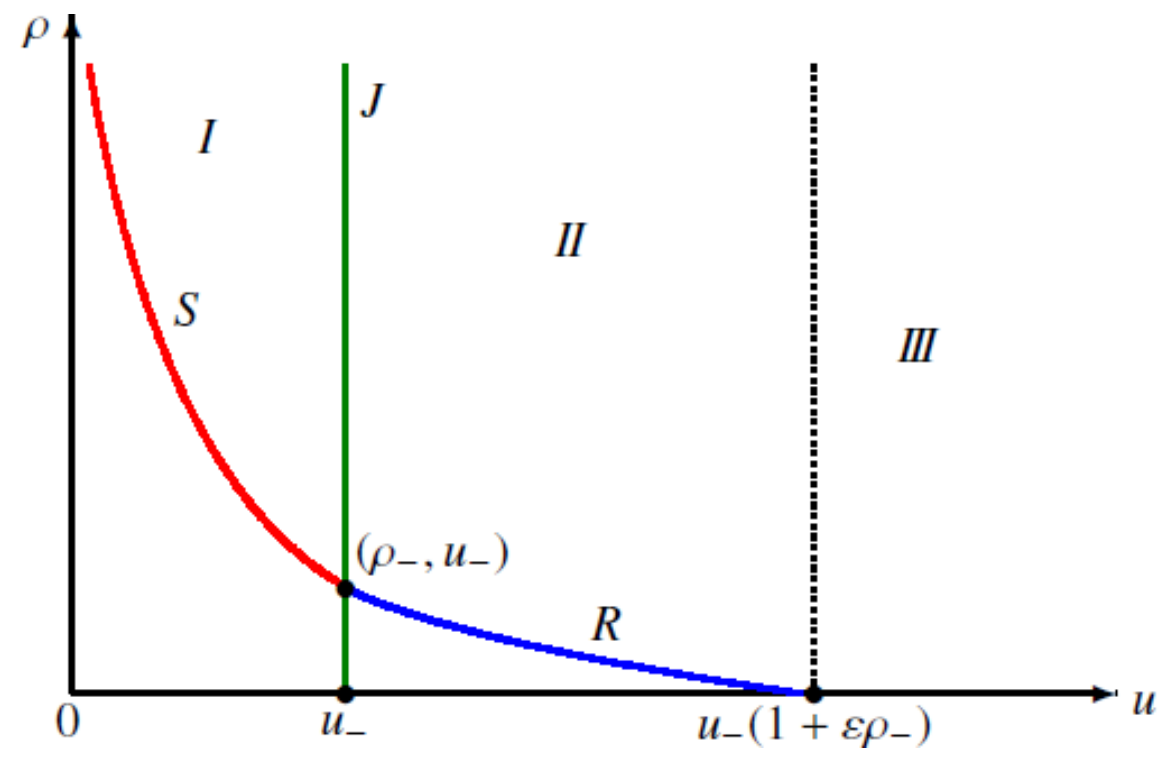




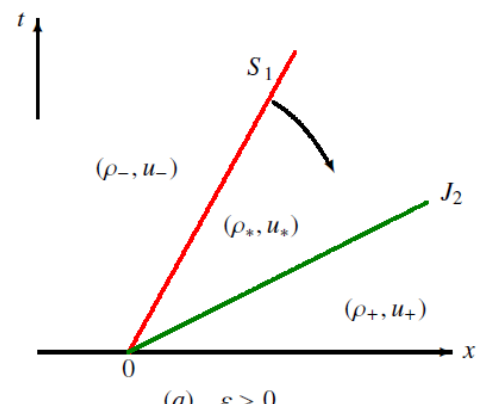

(a) $\varepsilon>0$
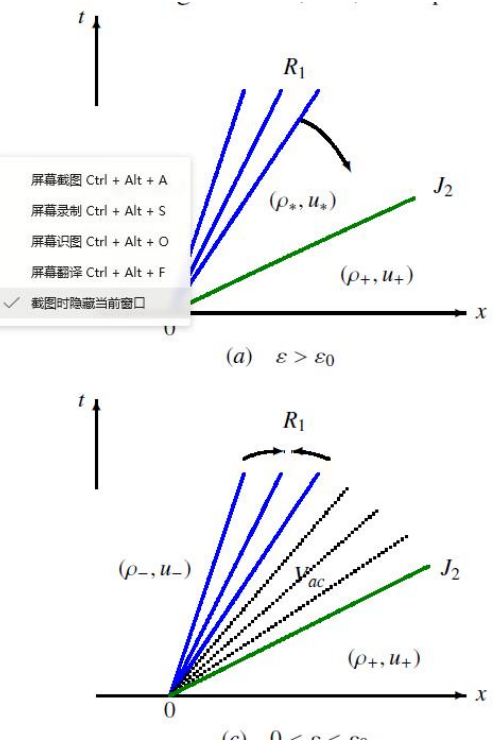

(c) $0<\varepsilon<\varepsilon_{0}$
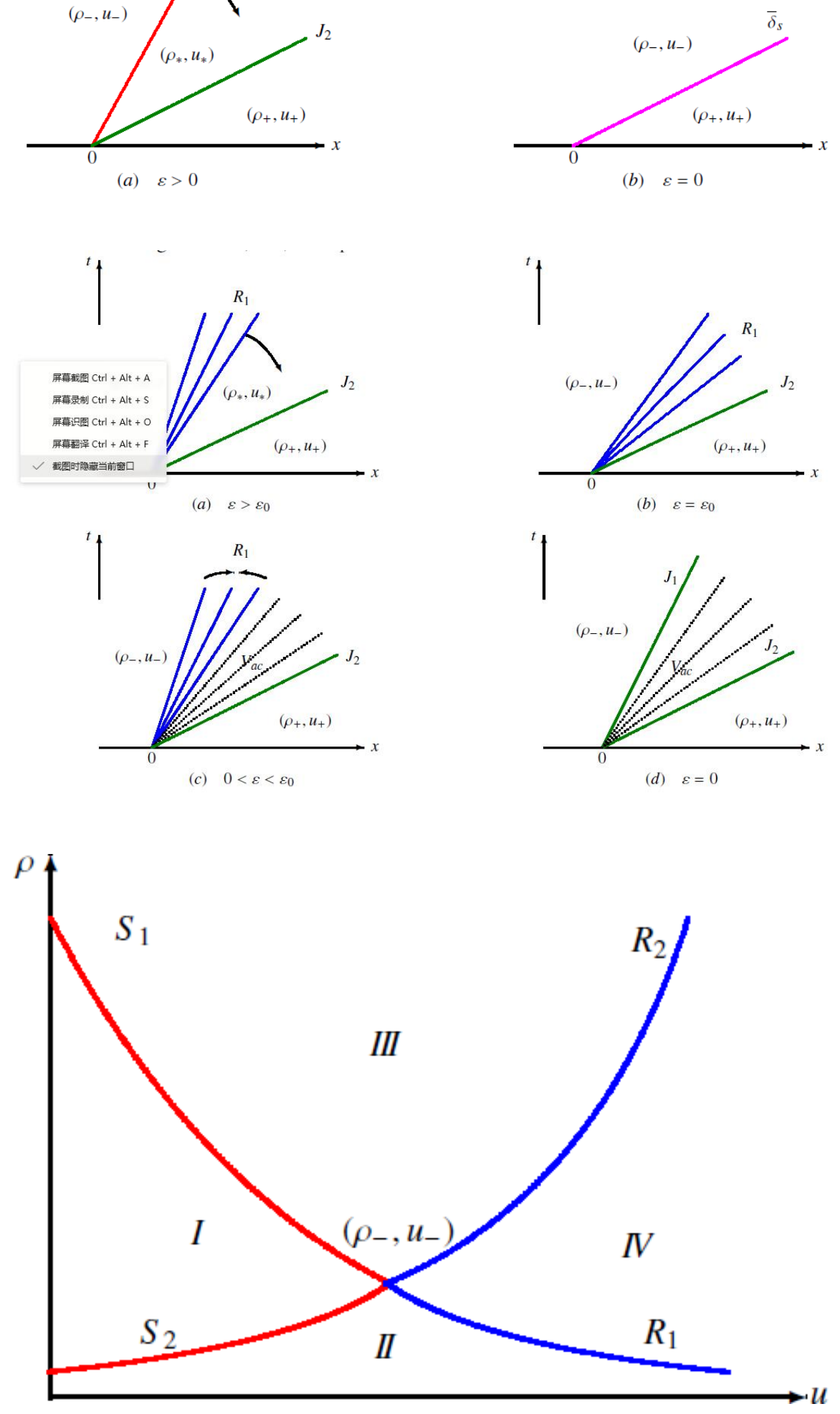

(d) $\varepsilon=0$ 

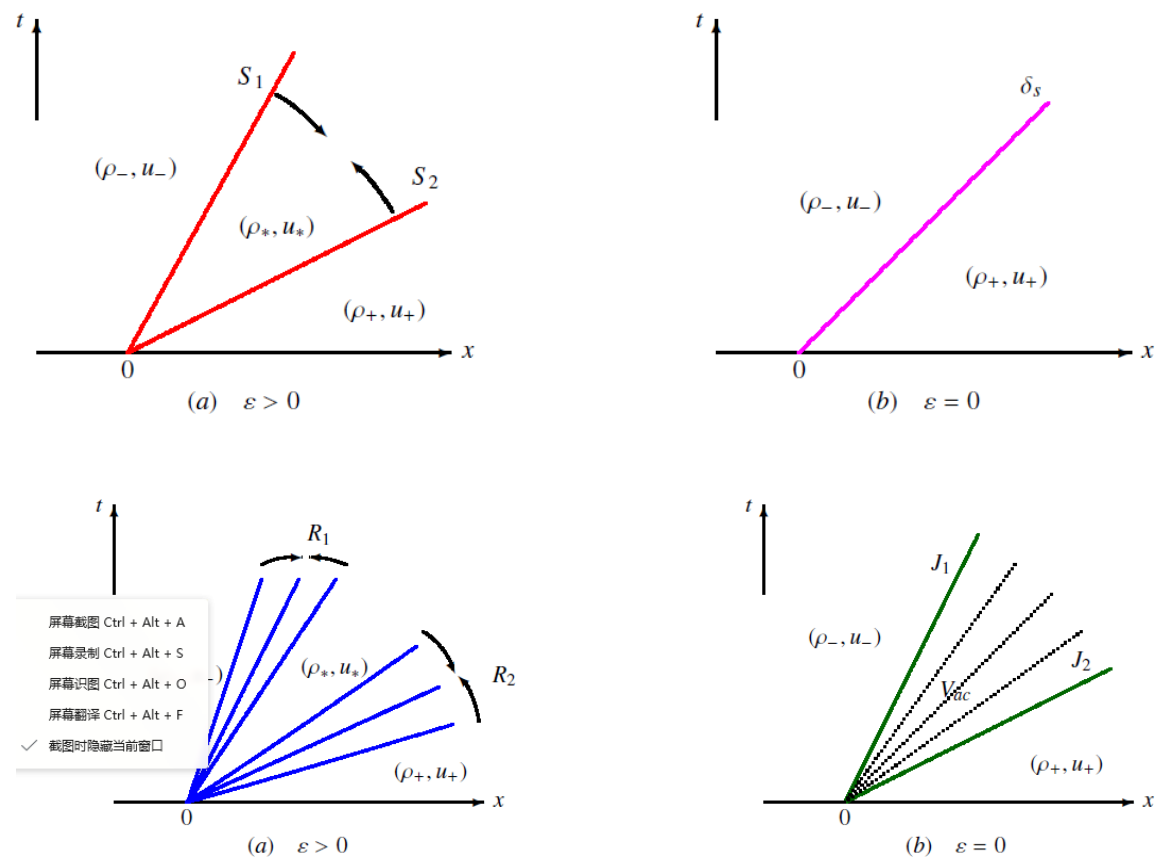